\title{
Robust maneuvering envelope estimation based on reachability analysis in an optimal control formulation*
}

\author{
Thomas Lombaerts ${ }^{1}$ and Stefan Schuet ${ }^{2}$ and Kevin Wheeler ${ }^{3}$ and Diana Acosta ${ }^{3}$ and John Kaneshige ${ }^{3}$
}

\begin{abstract}
This paper discusses an algorithm for estimating the safe maneuvering envelope of damaged aircraft. The algorithm performs a robust reachability analysis through an optimal control formulation while making use of time scale separation and taking into account uncertainties in the aerodynamic derivatives. Starting with an optimal control formulation, the optimization problem can be rewritten as a HamiltonJacobi-Bellman equation. This equation can be solved by level set methods. This approach has been applied on an aircraft example involving structural airframe damage. Monte Carlo validation tests have confirmed that this approach is successful in estimating the safe maneuvering envelope for damaged aircraft.
\end{abstract}

\section{INTRODUCTION}

All transportation systems need to focus on safety, but this applies especially to civil aviation. Therefore, in civil aviation, many developments focus on improving safety levels and reducing the risks that critical failures occur. In a recent study by CAST/ICAO, it can be observed that "loss of control in flight" (LOC-I) is the most frequent primary accident cause. This study is based on a statistical analysis of aircraft accidents between 2002 and 2011, and indicates that this category accounts for as much as $23 \%$ of all fatal aircraft accidents and involves most fatalities[1]. Benefit can be gained by developing technology which prevents these LOC-I accidents. From a flight dynamics point of view, with the technology and computing power available on this moment, it might have been possible to recover some of the aircraft in the accident category described above on the condition that non-conventional control strategies would have been applied. These non-conventional control strategies involve the so-called concept of fault tolerant flight control (FTFC), where the control system is capable of detecting and adapting to changes in the aircraft behaviour.

One FTFC strategy option is using a model based control routine. Previous research focused on a physical model approach[2]. In this setup, experiments have shown that not

\footnotetext{
*This work is supported by a Marie Curie International Outgoing Fellowship (IOF) within the 7th European Community Framework Program.

${ }^{1} \mathrm{~T}$. Lombaerts is with the German Aerospace Center DLR, Institute of System Dynamics and Control, Department of Aircraft Systems Dynamics, 82234 Weßling - Oberpfaffenhofen, Germany. Currently, he is Marie Curie Fellow and visiting researcher at NASA Ames Research Center, Intelligent Systems Division, Adaptive Control and Evolvable Systems (ACES) Group, Moffett Field, CA94035, USA. thomas.lombaerts@dlr.de and thomas.1ombaerts@nasa.gov

${ }^{2} \mathrm{~S}$. Schuet is with NASA Ames Research Center, Intelligent Systems Division, Physics based Methods Group, Moffett Field, CA94035, USA.

${ }^{3} \mathrm{~K}$. Wheeler and D. Acosta and J. Kaneshige are with NASA Ames Research Center, Intelligent Systems Division, Adaptive Control and Evolvable Systems (ACES) Group, Moffett Field, CA94035, USA.
}

only a reconfiguring controller is needed, but also some form of flight envelope protection, which prevents the airplane from leaving the safe flight envelope and losing control in flight[2]. The main challenge in this context is determining the new bounds of the safe flight envelope after failure, which are then used by the envelope protection algorithm[3].

Alternative and complementary research approaches for the purpose of loss of control prevention and prediction are among others passive adaptive control[4], data-based predictive control[5] and real-time optimal envelope limit estimation[6].

Determination of the flight envelope has been done in the literature through various methods. The most straightforward methods include wind tunnel testing, flight test experiments and high-fidelity model-based computation of attainable equilibrium sets or achievable trim points[7], [8], [9]. More complex methods include formulating flight envelope estimation as a reachability problem and solving this with level set methods and Hamilton-Jacobi equations [10], [11], [13], [14], [15], possibly with time scale separation [16] or semi-Lagrangian level sets [18]. Alternative methods rely on linearization and region of attraction analysis [19], determining controllability/maneuverability limits in a quaternionbased control architecture[20] or robustness analysis for determination of reliable flight regimes [21]. An approach suggested by Boeing, as part of the NASA program Dynamic Flight Envelope Assessment and Prediction (DFEAP), uses Control-Centric Modeling, dynamic flexible structure and load models [22]. In the frequency domain, stability margins can be estimated in real time via nonparametric system identification [23]. More focused techniques inspired by flight dynamics exist as well, such as determining the minimum lateral control speed[24].

From the perspective of the physical approach, the preferred interpretation of the safe maneuvering envelope considers reachability from the trim envelope. The stable and controllable trim envelope is considered an a-priori safe set. The backwards reachable set is defined as the set of states from where the trim envelope can be reached. The forwards reachable set is the set of states which can be reached from the trim envelope. Then the safe maneuvering flight envelope is the cross section of the forwards and backwards reachable sets. This interpretation is illustrated in Fig. 1. In addition, the backwards reachable set is the survivable flight envelope. After an upset due to damage, turbulence, a wake encounter etc., one can bring the aircraft back to a safe trim condition if the current flight condition is situated inside the backwards reachable set. 


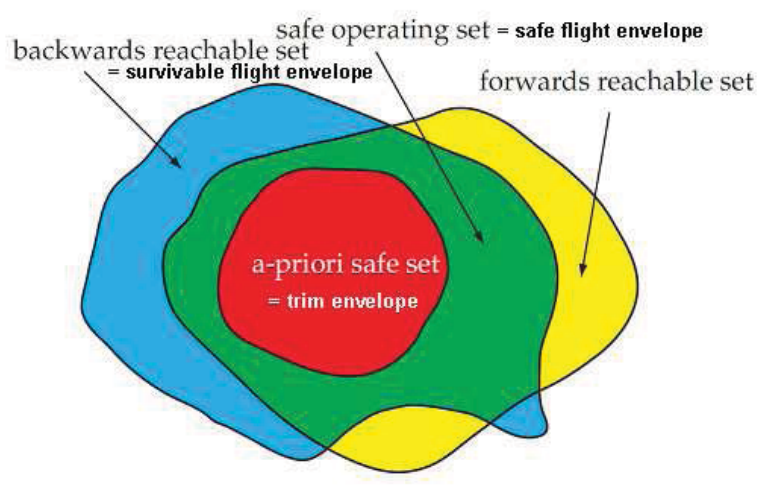

Fig. 1. Safe maneuvering envelope as intersection between forwards and backwards reachability, modified from source: van Oort[18]

The aim is to perform a combined forward and backward reachability analysis from the trim envelope as efficiently as possible, for on-line implementations. Based on previous research[6], level set methods are an excellent candidate. Two of the major challenges are the computational load and how to cope with nonlinear systems with higher dimensions. In general, an increase in technology readiness level (TRL) is envisaged.

Nonlinear systems with higher dimensions can be simplified by considering the principle of time scale separation[16]. The structure of time scale separation is analogous as applied for the fault tolerant control algorithm developed earlier[2]. The overview can be found in Fig. 2, which illustrates that a nine dimensional nonlinear problem is decoupled in three consecutive three dimensional optimization problems.

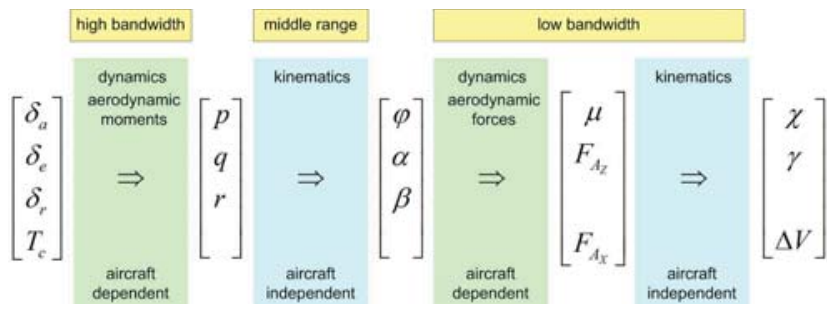

Fig. 2. Separation of dynamics over high bandwidth, middle range and low bandwidth. $F_{A_{X}}$ and $F_{A_{Z}}$ are defined in Eq. (9) and (10).

\section{OPTIMAL CONTROL FORMULATION}

It has been shown in the literature that maneuvering envelope estimation through reachability can be reformulated in the optimal control framework[10]. Consider a continuous time control system:

$$
\dot{\mathbf{x}}=\mathbf{f}(\mathbf{x}, \mathbf{u}, \Delta)
$$

with $\mathbf{x} \in \mathbb{R}^{n}, \mathbf{u} \in U \subseteq \mathbb{R}^{m}, \Delta \in D \subseteq \mathbb{R}^{k}, \mathbf{f}(\cdot, \cdot): \mathbb{R}^{n} \times U \rightarrow \mathbb{R}^{n}$, a function:

$$
l(\cdot): \mathbb{R}^{n} \rightarrow \mathbb{R}
$$

and an arbitrary time horizon $T \geq 0$. Let $\mathscr{U}_{\left[t, t^{\prime}\right]}$ denote the set of Lebesgue and bounded measurable functions from the interval $\left[t, t^{\prime}\right]$ to the set of admissible input values $U$. Define $\phi(\tau, t, \mathbf{x}, \mathbf{u}(\cdot), \Delta)$ as the state trajectory. $\Delta$ are defined as parameter uncertainties inside the predefined set of expected uncertainty values $D$. Given a set of states $K \subseteq \mathbb{R}^{n}$, the reachability question can be naturally formulated regarding the relation between the set $K$ and the state trajectories $\phi$ of Eq. (1) over the horizon $T$. Problem of interest is the following:

Robust reachability: Does there exist a $\mathbf{u} \in \mathscr{U}_{[0, T]}$ and a $t \in[0, T]$ such that the trajectory $\phi$ of the state $\mathbf{x}$ satisfies $\mathbf{x} \in K$, irrespective of $\Delta$ ?

The optimization problem can be formulated as a pursuit evasion game over the horizon $T \geq 0$ with target set $K \subseteq \mathbb{R}^{n}$ [17]. It is assumed that $\mathbf{u}$ is trying to bring or keep the state in the set $K$, whereas $\Delta$ is trying to drive it out of $K$. To ensure the game is well-posed, u is restricted to play non-anticipative strategies with respect to the unknown uncertainties $\Delta$.

For the types of safety problems considered here, a set of initial states has to be established such that $\mathbf{u}$ can win the game, in other words the set $\mathscr{R}$ can be characterized:

$$
\begin{aligned}
\mathscr{R}_{\Delta}(t, K)= & \left\{\mathbf{x} \in \mathbb{R}^{n} \mid \forall \Delta \in D, \exists \mathbf{u} \in \mathscr{U}_{[t, T]},\right. \\
& \exists \tau \in[t, T], \phi(\tau, t, \mathbf{x}, \mathbf{u}(\cdot), \Delta) \in K\}
\end{aligned}
$$

As done elsewhere in the literature[10], the characterization of this set can be done according to the principle of duality:

$$
\mathscr{R}(t, K)=\left(\mathscr{I}\left(t, K^{c}\right)\right)^{c}
$$

where $\bullet^{c}$ stands for the complement of $\bullet$. Through this principle, it can be characterized as an INFMIN problem[10]. The crux is to include the $\Delta$ 's as disturbances in the optimization function, they oppose the optimization over $\mathbf{u}$. Consider a closed set $K$, that can be written as the level set of a continuous function $l: \mathbb{R}^{n} \rightarrow \mathbb{R}$, i.e. $K=\left\{\mathbf{x} \in \mathbf{R}^{n} \mid l(\mathbf{x}) \geqslant 0\right\}$. As a consequence, the Invariance optimization formulation becomes[16]:

$$
\mathscr{I}(t, K)=\left\{\mathbf{x} \in \mathbb{R}^{n} \mid V_{1}(\mathbf{x}, t) \geqslant 0\right\}
$$

with:

$$
V_{1}(\mathbf{x}, t)=\inf _{\mathbf{u}(\cdot) \in \mathscr{U}[t, T]} \sup _{\Delta \in D} \min _{\tau \in[t, T]} l(\phi(\tau, t, \mathbf{x}, \mathbf{u}(\cdot), \Delta))
$$

This can be reformulated into an Hamilton-Jacobi-Bellmann Partial Differential Equation (HJB PDE)[10], [17]:

$$
\frac{\partial V_{1}}{\partial t}(\mathbf{x}, t)+\min _{\tau \in[t, T]}\left\{\inf _{\mathbf{u}(\cdot) \in \mathscr{U}[t, T]} \sup _{\Delta \in D} \frac{\partial V_{1}}{\partial \mathbf{x}}(\mathbf{x}, t) \mathbf{f}(\mathbf{x}, \mathbf{u}, \Delta)\right\}=0
$$

where $V_{1}(\mathbf{x}, T)=l(\mathbf{x})$ holds for backward integration and $V_{1}(\mathbf{x}, t)=l(\mathbf{x})$ applies to forward integration. These HJB PDE's can be solved by level sets, for which a toolbox is available in Matlab $^{\circledR}[11]$. 


\section{APPROACH}

The approach to calculate the safe maneuvering envelope after damage is based on the following steps:

- Identify the updated aircraft parameters after damage (not discussed here, see [12]). This concerns primarily estimating new post-damage values for the aerodynamic derivatives such as $C_{L_{0}}, C_{L_{\alpha}}, C_{D_{0}}, C_{D_{\alpha}}, C_{D_{\alpha^{2}}}$ and $C_{Y_{\beta}}$ together with their uncertainty bounds.

- Calculate the post-damage trim envelope based on the updated aircraft parameters (not discussed here, see [12]).

- Based on the previous step, define reference trim boundaries for airspeed $V$, flight path angle $\gamma$ and bank angle $\varphi$ as well as grid step size $\Delta V, \Delta \gamma$ and $\Delta \varphi$.

- Define an implicit function accordingly over $V$ and $\gamma$. This needs to be done for every value of $\varphi$ in case speed and flight path angle are bank angle dependent, i.e. $V=V(\varphi)$ and $\gamma=\gamma(\varphi)$.

- The Level sets method toolbox[11] relies on the Hamiltonian in Eq. (6) as a gradient to evolve the implicit function and thus reference boundaries over time

- The cost function $V_{1}$ becomes a three dimensional functions, where cross sections reflect the situation for a specific time instant $t_{a}$.

- A dissipation function is needed to guarantee numerical stability during these calculations. As a consequence, slightly conservative results are obtained for the boundaries, but analysis has shown that this dissipation has a minor effect on the results. In this specific context, the chosen dissipation function is a Lax Friedrichs dissipation function[11]

\section{APPLICATION EXAMPLE}

To illustrate how the envelope estimating algorithm works, a nonlinear 3D aircraft example is considered. At this point, only the slow dynamics as specified in Fig. 2 are considered. Future work will extend to the faster dynamics. The data used in this example are based on the RCAM (Research Civil Aircraft Model) simulation model[25]. The acting forces on the aircraft are illustrated in Fig. 3 for a symmetric flight condition.

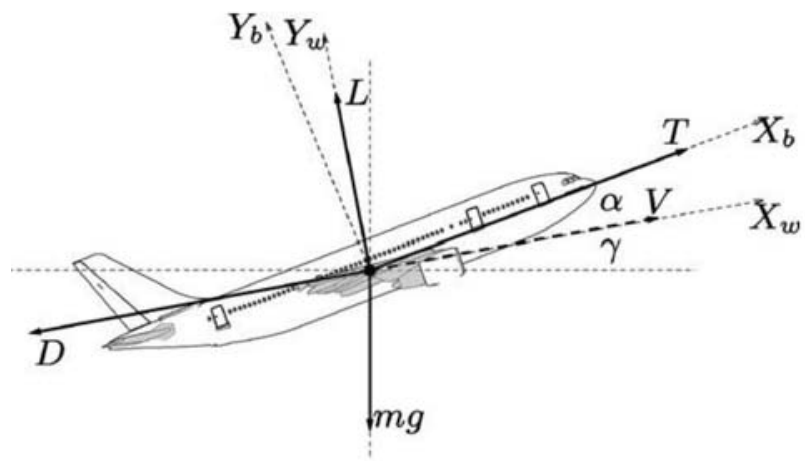

Fig. 3. Acting forces on the aircraft model, source: Lygeros[10]
For the complete 3D situation, the equations of motion for $\dot{V}$ and $\dot{\gamma}$ are written as follows[2]:

$$
\begin{aligned}
F_{A_{X}}-W \sin \gamma & =m \dot{V} \\
F_{A_{Z}} \cos \varphi+F_{A_{Y}} \sin \varphi+W \cos \gamma & =-m V \dot{\gamma}
\end{aligned}
$$

Where the aerodynamic force components can be simplified assuming small aerodynamic angles $\alpha$ and $\beta^{1}$.

$$
\begin{aligned}
& F_{A_{X}}=T \cos \beta \cos \alpha-D(V, \alpha) \approx T-D(V, \alpha) \\
& F_{A_{Z}}=-T \sin \alpha-L(V, \alpha) \approx-L(V, \alpha) \\
& F_{A_{Y}}=-T \sin \beta \cos \alpha+Y_{\text {aero }}(V, \beta) \approx Y_{\text {aero }}(V, \beta)
\end{aligned}
$$

with the following expansions for lift $L$, drag $D$ and sideforce $Y_{\text {aero }}$ :

$$
\begin{aligned}
D(V, \alpha) & =\bar{q} S\left(C_{D_{0}}+C_{D_{\alpha}} \alpha+C_{D_{\alpha^{2}}} \alpha^{2}\right) \\
L(V, \alpha) & =\bar{q} S\left(C_{L_{0}}+C_{L_{\alpha}} \alpha\right) \\
Y_{\text {aero }}(V, \beta) & =\bar{q} S\left(C_{Y_{\beta}} \beta\right)
\end{aligned}
$$

where the dynamic pressure $\bar{q}=1 / 2 \rho V^{2}$. Currently, these conventional expansions are used in this approach. However, for future work, more elaborate expansions can be relied on, e.g. where drag $D$ also depends on the absolute value of the sideslip angle $|\beta|$.

The corresponding numerical values are: $m=120 \cdot 10^{3} \mathrm{~kg}$, $g=9.81 \mathrm{~m} / \mathrm{s}^{2}, W=m g, \rho=1.225 \mathrm{~kg} / \mathrm{m}^{3}$ (sea level), $S=$ $260 \mathrm{~m}^{2}, C_{L_{0}}=1.0656, C_{L_{\alpha}}=6.0723, C_{D_{0}}=0.1599, C_{D_{\alpha}}=$ $0.5035, C_{D_{\alpha^{2}}}=2.1175, C_{Y_{\beta}}=-1.6$.

In the perspective of reachability from stable and controllable trim conditions, the primary states of interest are airspeed $V$ and flight path angle $\gamma$. Considering time scale separation as presented in Fig. 2, the virtual inputs for the slow dynamics are roll angle $\varphi$, angle of attack $\alpha$, sideslip angle $\beta$ and thrust $T$. This framework and combining Eqs. (7)-(14) allows to define the general dynamics $\mathbf{f}$ in Eq. (1) by the following differential equation:

$$
\begin{aligned}
{\left[\begin{array}{c}
\dot{V} \\
\dot{\gamma}
\end{array}\right]=\left[\begin{array}{c}
-\frac{\rho S}{2 m} V^{2} C_{D_{0}}-g \sin \gamma \\
-\frac{g}{V} \cos \gamma
\end{array}\right]+} \\
+\left[\begin{array}{c}
\cos \alpha \cos \beta \\
(\cos \varphi \sin \alpha \cos \beta-\sin \varphi \sin \beta) \frac{1}{V}
\end{array}\right] \frac{T}{m}+ \\
+\left[\begin{array}{c}
-\frac{\rho S}{2 m} V^{2}\left(C_{D_{\alpha}} \alpha+C_{D_{\alpha^{2}}} \alpha^{2}\right) \\
\frac{\rho S}{2 m} V\left(C_{L_{0}}+C_{L_{\alpha}} \alpha\right) \cos \varphi
\end{array}\right]+\left[\begin{array}{c}
0 \\
-\frac{\rho S}{2 m} V C_{Y_{\beta}} \beta \sin \varphi
\end{array}\right]
\end{aligned}
$$

Assuming small aerodynamic angles, as earlier, simplifies the differential equation:

$$
\begin{aligned}
{\left[\begin{array}{c}
\dot{V} \\
\dot{\gamma}
\end{array}\right] } & =\left[\begin{array}{c}
-\frac{\rho S}{2 m} V^{2} C_{D_{0}}-g \sin \gamma \\
-\frac{g}{V} \cos \gamma
\end{array}\right]+\left[\begin{array}{c}
\frac{1}{m} \\
0
\end{array}\right] T+ \\
+ & {\left[\begin{array}{c}
-\frac{\rho S}{2 m} V^{2}\left(C_{D_{\alpha}} \alpha+C_{D_{\alpha^{2}}} \alpha^{2}\right) \\
\frac{\rho S}{2 m} V\left(C_{L_{0}}+C_{L_{\alpha}} \alpha\right) \cos \varphi
\end{array}\right]+\left[\begin{array}{c}
0 \\
-\frac{\rho S}{2 m} V C_{Y_{\beta}} \beta \sin \varphi
\end{array}\right] }
\end{aligned}
$$

\footnotetext{
${ }^{1}$ Note that the allowed ranges in this specific example are set at $\alpha \in$ $\left[0^{\circ} ; 14.5^{\circ}\right], \beta \in\left[-5^{\circ} ; 5^{\circ}\right]$, as defined later. The small angle assumption holds up to $\pm 30^{\circ}$.
} 
This results in a Hamiltonian function with decoupled virtual inputs $T, \alpha$ and $\beta$. Roll angle $\varphi$ is not decoupled, but will be treated as a discretely gridded input. This decoupling significantly promotes computational efficiency, which is crucial for on-line applications. The Hamiltonian function becomes:

$$
\begin{aligned}
H(\mathbf{p}, \mathbf{x}, \mathbf{u}) & =\frac{p_{1}}{m} T-p_{1} \frac{\rho S}{2 m} V^{2} C_{D_{\alpha^{2}}} \alpha^{2}+ \\
& +\frac{\rho S}{2 m} V\left(p_{2} C_{L_{\alpha}} \cos \varphi-p_{1} V C_{D_{\alpha}}\right) \alpha+ \\
& -p_{2} \frac{\rho S}{2 m} V C_{Y_{\beta}} \sin \varphi \beta
\end{aligned}
$$

where $\mathbf{p}$ are the co-states of the value function: $p_{1}=\frac{\partial V_{1}}{\partial V}$ and $p_{2}=\frac{\partial V_{1}}{\partial \gamma}$. This Hamiltonian is linear in thrust $T$ and sideslip angle $\beta$ and quadratic in angle of attack $\alpha$. This structure allows for an efficient optimization routine over the inputs. The trim envelope boundaries are: $V_{\min }=60 \mathrm{~m} / \mathrm{s}, V_{\max }=100 \mathrm{~m} / \mathrm{s}$ and $\gamma_{\min / \max }= \pm 10^{\circ}$. The allowed ranges of the virtual inputs are: $T_{\min }=20546 N, T_{\max }=410920 N, \alpha_{\min }=0^{\circ}, \alpha_{\max }=$ $14.5^{\circ}$ (no stall), $\phi_{\min / \max }= \pm 60^{\circ}, \beta_{\min / \max }= \pm 5^{\circ}$. The maximizers $\hat{T}, \hat{\alpha}$ and $\hat{\beta}$ depend on the sign of the costates $p_{1}$ and $p_{2}$. Recall that $V>0, C_{D_{\alpha}}>0, C_{D_{\alpha^{2}}}>0, C_{L_{\alpha}}>0, C_{Y_{\beta}}<$ 0 . Due to the underlying physics, no sign changes for these parameters are to be expected in case of structural changes. Define $\hat{p}=\frac{p_{2} C_{L_{\alpha}} \cos \varphi-p_{1} V C_{D \alpha}}{2 p_{1} V C_{D} \alpha^{2}}$ and $\bar{\alpha}=\frac{\alpha_{\min }+\alpha_{\max }}{2}$. Then the optimizing control inputs can be defined for invariance:

- If $p_{1}>0$ then $\hat{T}=T_{\min }$ and

if $\hat{p}>\bar{\alpha}$ then $\hat{\alpha}=\alpha_{\min }$

if $\hat{p}=\bar{\alpha}$ then $\hat{\alpha}=\alpha_{\min }$ or $\alpha_{\max }$

if $\hat{p}<\bar{\alpha}$ then $\hat{\alpha}=\alpha_{\max }$

- If $p_{1}=0$ then $\hat{T} \in\left[T_{\min } ; T_{\max }\right]$ and

if $p_{2}>0$ then $\hat{\alpha}=\alpha_{\min }$

if $p_{2}=0$ then $\hat{\alpha} \in\left[\alpha_{\min } ; \alpha_{\max }\right]$

if $p_{2}<0$ then $\hat{\alpha}=\alpha_{\max }$

- If $p_{1}<0$ then $\hat{T}=T_{\max }$ and

if $\hat{p} \leq \alpha_{\min }$ then $\hat{\alpha}=\alpha_{\text {min }}$

if $\alpha_{\min } \leq \hat{p} \leq \alpha_{\max }$ then $\hat{\alpha}=\hat{p}$

if $\hat{p} \geq \alpha_{\max }$ then $\hat{\alpha}=\alpha_{\max }$

- if $p_{2} \sin \varphi>0$ then $\hat{\beta}=\beta_{\text {min }}$

- if $p_{2} \sin \varphi=0$ then $\hat{\beta} \in\left[\beta_{\min } ; \beta_{\max }\right]$

- if $p_{2} \sin \varphi<0$ then $\hat{\beta}=\beta_{\max }$

For the purpose of maximizing the cost function with respect to the uncertainties $\Delta$, the Hamiltonian from Eq. (17) can be rewritten, this time including parts independent of the inputs $T, \alpha$ or $\beta$ but with some aerodynamic derivative(s):

$$
\begin{aligned}
H(\mathbf{p}, \mathbf{x}, \mathbf{u}) & =-p_{1} \frac{\rho S}{2 m} V^{2} C_{D_{0}}+p_{2} \frac{\rho S}{2 m} V C_{L_{0}} \cos \varphi+ \\
& -p_{1} \frac{\rho S}{2 m} V^{2}\left(C_{D_{\alpha}} \alpha+C_{D_{\alpha^{2}}} \alpha^{2}\right)+ \\
& +p_{2} V \frac{\rho S}{2 m} C_{L_{\alpha}} \cos \varphi \alpha-p_{2} \frac{\rho S}{2 m} V C_{Y_{\beta}} \sin \varphi \beta
\end{aligned}
$$

It can be observed that the aerodynamic derivatives all appear linearly in an uncoupled way, which allows a similar procedure to solve the optimization as previously. By rewriting the
Hamiltonian as a summation of terms, where each term is a multiplication of a variable involving a costate, a constant factor and a derivative, one can determine the sign of this factor, which consists of the predefined physical parameters:

$$
\begin{aligned}
H(\mathbf{p}, \mathbf{x}, \mathbf{u}) & =-p_{1} \underbrace{\frac{\rho S}{2 m} V^{2}}_{>0} C_{D_{0}}+p_{2} \underbrace{\frac{\rho S}{2 m} V \cos \varphi C_{L_{0}}}_{>0}+ \\
& -p_{1} \underbrace{\frac{\rho S}{2 m} V^{2} \alpha^{2}}_{>0} C_{D_{\alpha^{2}}}+p_{2} \underbrace{\frac{\rho S}{2 m} V \alpha \cos \varphi}_{>0} C_{L_{\alpha}}+ \\
& -p_{1} \underbrace{\frac{\rho S}{2 m} V^{2} \alpha}_{>0} C_{D_{\alpha}}-p_{2} \sin \varphi \beta \underbrace{\frac{\rho S}{2 m} V C_{Y_{\beta}}}_{>0}
\end{aligned}
$$

where it should be noted that $\varphi \in\left[-60^{\circ} ; 60^{\circ}\right], \alpha \in$ $\left[0^{\circ} ; 14.5^{\circ}\right], \beta \in\left[-5^{\circ} ; 5^{\circ}\right]$. Furthermore airspeed $V>0$ and for the aerodynamic derivatives, it is known that $C_{D_{\alpha}}>0, C_{D_{\alpha^{2}}}>$ $0, C_{L_{\alpha}}>0, C_{Y_{\beta}}<0$. Due to the underlying physics, no sign changes for these parameters are to be expected in case of uncertainty or structural changes.

Based on this formulation, optimal control inputs for the aerodynamic derivatives can be defined as given in Table I where $\bar{C}_{\bullet}=C_{\bullet_{\text {max }}}$ and $\underline{C}_{\bullet}=C_{\bullet_{\text {min }}}$.

With this information, it is possible to create an entire "uncertainty band" around the envelope, however, here focus will be placed on the "worst-case" minimal size envelope.

Fig. 4 compares the 3D envelopes with and without uncertainty, where two levels of uncertainty have been considered here, namely $10 \%$ and $20 \%$ of uncertainty on all aerodynamic derivatives. For the purpose of this example, identical ratios of standard deviations over nominal values have been defined for all derivatives, but the algorithm is capable to deal with individual standard deviations which can vary between the different aerodynamic derivatives. It can be clearly seen in Fig. 4 that larger degrees of uncertainty result in more significant shrinking of the envelope, since this is a "worst-case" minimal size envelope.

Fig. 5 analyzes the $V, \gamma$ maneuvering envelope for different values of bank angle $\varphi$, including robustness for uncertainties of $10 \%$ and 20\%. By comparing Fig. 5(a), 5(b) and 5(c), it can be seen that larger bank angles have an influence on the climb capability of the aircraft. This is due to the physical principle that climb capability of lift force is provided through $L \cos \varphi$, which confirms a smaller decrease for smaller bank angles (up to $\varphi=25^{\circ}$ as shown in Fig. 5(b)) but a much more significant change for larger bank angles as can be seen in Fig. 5(c).

\section{VALIDATION OF RESULTS}

The aforementioned results have been validated by means of Monte Carlo analyses. For this purpose, different bangbang input signals have been inserted in the system. The extreme values of these signals correspond with the range limits. The time instant for the step change and the initial value for the input (maximum or minimum) vary over the Monte Carlo analysis. Running a nonlinear simulation of the 
TABLE I

OPTIMAL CONTROL INPUTS FOR ROBUSTNESS AGAINST UNCERTAIN AERODYNAMIC DERIVATIVES

\begin{tabular}{ccc}
\hline \hline sign of costate & minimizer & maximizer \\
\hline$p_{1} \geq 0$ & $\hat{C}_{D_{0}}=\bar{C}_{D_{0}}, \hat{C}_{D_{\alpha}}=\bar{C}_{D_{\alpha}}, \hat{C}_{D_{\alpha^{2}}}=\bar{C}_{D_{\alpha^{2}}}$ & $\hat{C}_{D_{0}}=\underline{C}_{D_{0}}, \hat{C}_{D_{\alpha}}=\bar{C}_{D_{\alpha}}, \hat{C}_{D_{\alpha^{2}}}=\underline{C}_{D_{\alpha^{2}}}$ \\
$p_{1}<0$ & $\hat{C}_{D_{0}}=\underline{C}_{D_{0}}, \hat{C}_{D_{\alpha}}=\underline{C}_{D_{\alpha}}, \hat{C}_{D_{\alpha^{2}}}=\underline{C}_{D_{\alpha^{2}}}$ & $\hat{C}_{D_{0}}=\bar{C}_{D_{0}}, \hat{C}_{D_{\alpha}}=\bar{C}_{D_{\alpha}}, \hat{C}_{D_{\alpha^{2}}}=\bar{C}_{D_{\alpha^{2}}}$ \\
$p_{2} \geq 0$ & $\hat{C}_{L_{0}}=\underline{C}_{L_{0}}, \hat{C}_{L_{\alpha}}=\underline{C}_{L_{\alpha}}$ & $\hat{C}_{L_{0}}=\bar{C}_{L_{0}}, \hat{C}_{L_{\alpha}}=\bar{C}_{L_{\alpha}}$ \\
$p_{2}<0$ & $\hat{C}_{L_{0}}=\bar{C}_{L_{0}}, \hat{C}_{L_{\alpha}}=\bar{C}_{L_{\alpha}}$ & $\hat{C}_{L_{0}}=\underline{C}_{L_{0}}, \hat{C}_{L_{\alpha}}=\underline{C}_{L_{\alpha}}$ \\
$p_{2} \sin \varphi \cdot \beta \geq 0$ & $\hat{C}_{Y_{\beta}}=\bar{C}_{Y_{\beta}}$ & $\hat{C}_{Y_{\beta}}=\underline{C}_{Y_{\beta}}$ \\
$p_{2} \sin \varphi \cdot \beta<0$ & $\hat{C}_{Y_{\beta}}=\underline{C}_{Y_{\beta}}$ & $\hat{C}_{Y_{\beta}}=\bar{C}_{Y_{\beta}}$ \\
\hline \hline
\end{tabular}

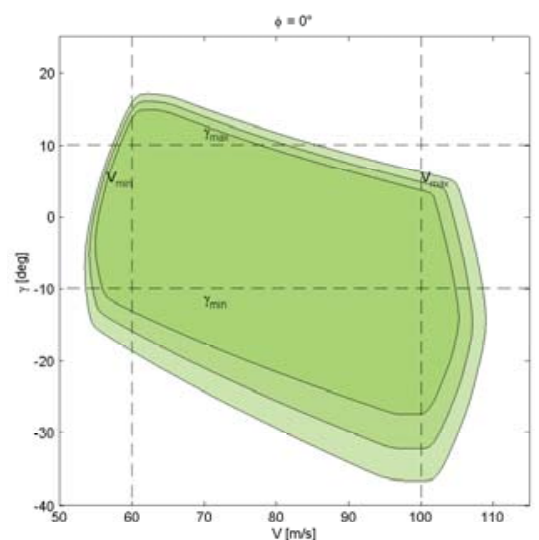

(a) $\varphi=0^{\circ}$

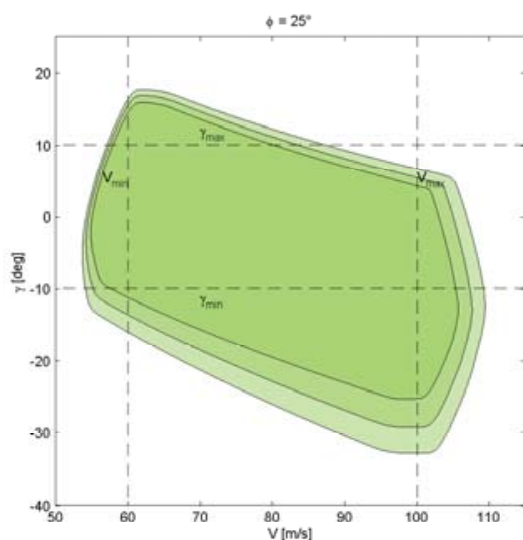

(b) $\varphi=25^{\circ}$

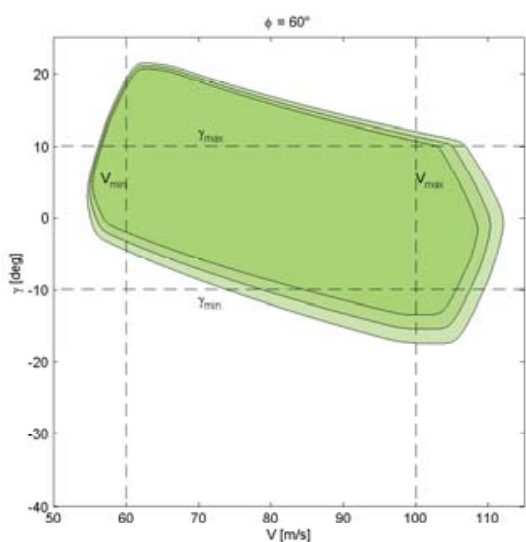

(c) $\varphi=60^{\circ}$

Fig. 5. $V, \gamma$ maneuvering envelope of RCAM model for time horizon $T=2 s$ for different bank angles and different uncertainty levels $(0 \%$, $10 \%$ and $20 \%$ uncertainty). Smaller envelope areas correspond to larger uncertainty bounds.

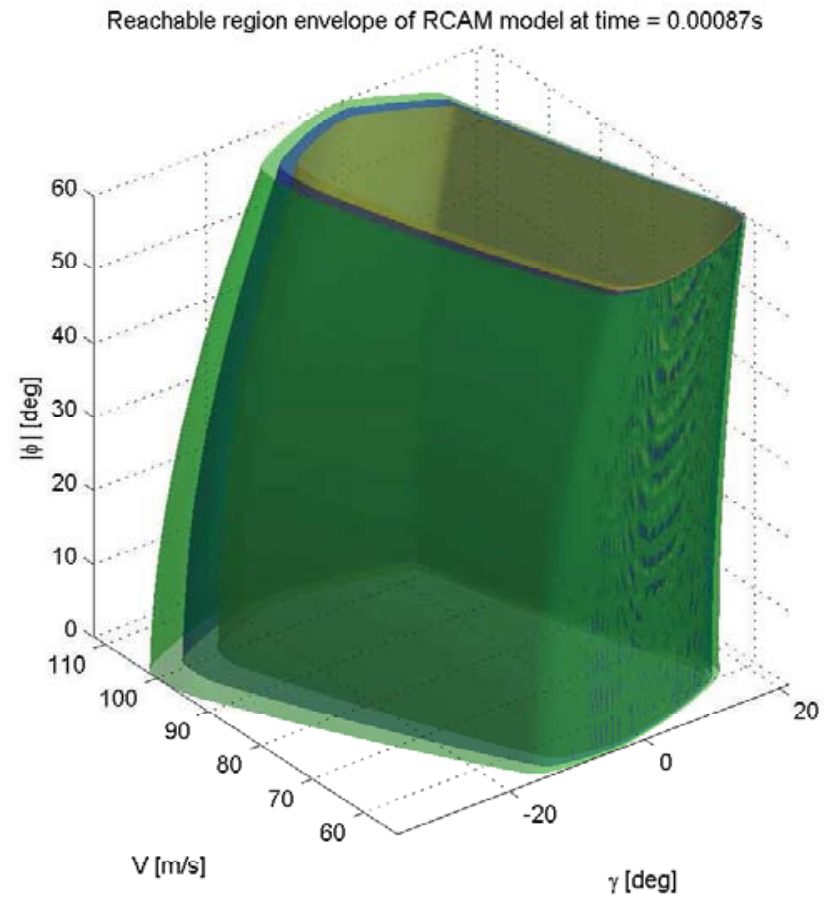

Fig. 4. Comparison of 3D envelopes with and without uncertainty: nominal (green), 10\% uncertainty (blue), 20\% uncertainty (yellow) aircraft model for the same time span as the time horizon $T=2 s$ in the reachability analysis and plotting the traces in the envelopes, results in Fig. 6. For initial conditions $\mathbf{x}_{0}$ within the backwards reachable set $\mathscr{R}(T=2 s, K)$, it is always possible to find at least one admissible input $\mathbf{u}(\cdot)$ which will bring part of the state trajectory $\phi(\tau, t, \mathbf{x}, \mathbf{u}, \Delta)$ towards the end point at $T=2 s$ inside the trim envelope $K$. On the other hand, from outside the backwards reachability set $\mathscr{R}(T=2 s, K)$, it is impossible for the state trajectory $\phi(\tau, t, \mathbf{x}, \mathbf{u}, \Delta)$ to reach the reference envelope $K$ within $T=$ $2 s$, independent from which input $\mathbf{u}$ is applied. Many more Monte Carlo validations have been performed for different initial conditions $\mathbf{x}_{0}$, which all confirm the accuracy of the envelope in a similar way as shown here. Moreover, these Monte Carlo analyses have been based on the non-simplified aircraft model. As such, it has been demonstrated that the simplifying assumption made in Eq. (16) is acceptable and does not significantly perturb the results.

\section{CONCLUSIONS}

In this paper, a computationally efficient algorithm for estimating the safe maneuvering envelope of damaged aircraft has been discussed. The algorithm performs a robust reachability analysis through an optimal control formulation while making use of time scale separation and taking into account uncertainties in the aerodynamic derivatives. The safe maneuvering envelope is defined as the cross section 

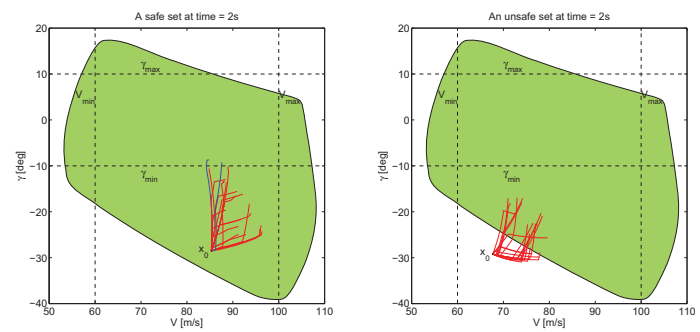

Fig. 6. Backwards reachability for RCAM model for time horizon $T=2 s$, including Monte Carlo Analysis

between the forwards reachable and backwards reachable sets, which have been calculated starting from the stable trim envelope. Moreover, the backwards reachable set can be considered as the survivable maneuvering envelope, from where it is possible to bring the aircraft back to a safe trim condition after an upset due to damage, turbulence, a wake encounter etc. Results were found to be consistent with the underlying physical principles. This approach differs from others since it is physically inspired. This more transparent approach allows interpreting data in each step, and it is assumed that the physics based approach will therefore facilitate certification for future real life applications.

\section{ACKNOWLEDGMENT}

The authors would like to acknowledge Kalmanje $\mathrm{Kr}$ ishnakumar, Nithin Govindarajan, Vahram Stepanyan and Jonathan Barlow for their important contributions to the research.

\section{REFERENCES}

[1] Statistical Summary of Commercial Jet Airplane Accidents - Worldwide Operations 1959 - 2011, Tech. rep., Boeing Commercial Aircraft, July 2012, Available at: http://www.boeing.com/news/techissues/pdf/statsum.pdf.

[2] Lombaerts, T., Fault Tolerant Flight Control - A Physical Model Approach, PhD dissertation, Delft University of Technology, May 2010 Available at: http://repository.tudelft.nl/view/ir/uuid\%3A538b0174fe84-43af-954d-02f256b2ec50/.

[3] Falkena, W., Borst, C., and Mulder, J., Investigation of Practical Flight Envelope Protection Systems for Small Aircraft, Guidance, Navigation, and Control and Co-located Conferences, No. AIAA 20107701, American Institute of Aeronautics and Astronautics, Aug. 2010.

[4] Stepanyan, V., Bijl, H., Krishnakumar, K., and Barlow, J., Adaptive Estimation Based Loss of Control Detection and Mitigation, Guidance, Navigation, and Control and Co-located Conferences, No. AIAA 20116609, American Institute of Aeronautics and Astronautics, Aug. 2011.

[5] Barlow, J., Stepanyan, V., and Krishnakumar, K., Estimating Lossof-Control: a Data-Based Predictive Control Approach, Guidance, Navigation, and Control and Co-located Conferences, No. AIAA 20116408, American Institute of Aeronautics and Astronautics, Aug. 2011.

[6] Govindarajan, N., An Optimal Control Approach for Estimating Aircraft Command Margin - with applications to Loss-Of-Control Prevention, Masters thesis, Delft University of Technology, October 2012, Available at: http://repository.tudelft.nl/view/ir/uuid\%3A48dbad61$728 \mathrm{a}-4 \mathrm{c} 7 \mathrm{e}-\mathrm{ba3e}$-cf8382ef1cef/.
[7] Tang, L., Roemer, M., Bharadwaj, S., and Belcastro, C., An Integrated Health Assessment and Fault Contingency Management System for Aircraft, AIAA Guidance, Navigation and Control Conference, No. AIAA 2008-6505, August 2008.

[8] Boskovic, J. D., Redding, J., and Knoebel, N., An Adaptive Fault Management (AFM) System for Resilient Flight Control, AIAA Guidance, Navigation and Control Conference, No. AIAA 2009-6263, August 2009.

[9] Kwatny, H. G. and Allen, R. C., Safe Set Maneuverability of Impaired Aircraft, Guidance, Navigation, and Control and Co-located Conferences, No. AIAA 2012-4405, American Institute of Aeronautics and Astronautics, Aug. 2012.

[10] Lygeros, J., On reachability and minimum cost optimal control, Automatica, Vol. 40, 2004, pp. 917-927.

[11] Mitchell, I. M., The flexible, extensible and efficient toolbox of level set methods, Journal of Scientific Computing, Vol. 35, 2008, pp. 300 329 .

[12] Schuet, S., Acosta, D., Wheeler, K., Kaneshige, J. and Lombaerts, T., An Adaptive Nonlinear Aircraft Maneuvering Envelope Estimation Approach for Online Applications, AIAA Guidance, Navigation and Control Conference, accepted for presentation, January 2014

[13] Kwatny, H. G., Dongmo, J.-E. T., Bor-Chin Chang, G. B., Yasar, M., and Belcastro, C., Aircraft Accident Prevention: Loss-of-Control Analysis, AIAA Guidance, Navigation and Control Conference, No. AIAA 2009-6256, August 2009.

[14] Tang, L., Roemer, M., Ge, J., Crassidis, A., Prasad, J., and Belcastro, C., Methodologies for Adaptive Flight Envelope Estimation and Protection, AIAA Guidance, Navigation and Control Conference, No. AIAA 2009-6260, August 2009.

[15] Allen, R. C., Kwatny, H. G., and Bajpai, G., Safe Set Protection and Restoration for Unimpaired and Impaired Aircraft, Guidance, Navigation, and Control and Co-located Conferences, No. AIAA 20124822, American Institute of Aeronautics and Astronautics, Aug. 2012.

[16] Kitsios, I. and Lygeros, J., Launch pad Abort Flight Envelope Computation for a Personnel Launch Vehicle using Reachability, AIAA Guidance, Control and Navigation, No. AIAA 2005-6150, August 2005.

[17] Kitsios, I. and Lygeros, J., Final Glide-back Envelope Computation for Reusable Launch Vehicle Using Reachability, IEEE Conference on Decision and Control, and the European Control Conference, pp. 4059 - 4064, December 2005.

[18] Oort, E. V., Chu, P., and Mulder, J. A., Advances in Aerospace Guidance, Navigation and Control, chap. Maneuvering Envelope Determination through Reachability Analysis, Springer - Verlag, 2011, pp. 91-102.

[19] Pandita, R., Chakraborty, A., Seiler, P., and Balas, G., Reachability and Region of Attraction Analysis applied to GTM Dynamic Flight Envelope Assessment, AIAA Guidance, Navigation and Control Conference, No. AIAA 2009-6258, August 2009.

[20] Bacon, B. J., Quaternion based control architecture for determining control/maneuverability limits, Guidance, Navigation, and Control and Co-located Conferences, No. AIAA 2012-5028, American Institute of Aeronautics and Astronautics, Aug. 2012.

[21] Shin, J.-Y. and Belcastro, C., Robustness Analysis and Reliable Flight Regime Estimation of an Integrated Resilient Control System for a Transport Aircraft, AIAA Guidance, Navigation and Control Conference, No. AIAA 2008-6656, August 2008.

[22] Urnes, J. M., Reichenbach, E. Y., and Smith, T. A., Dynamic Flight Envelope Assessment and Prediction, AIAA Guidance Navigation and Control Conference, No. AIAA 2008-6983, August 2008.

[23] Lichter, M. D., Bateman, A. J., and Balas, G., Flight Test Evaluation of a Run-time Stability Margin Estimation Tool, AIAA Guidance, Navigation and Control Conference, No. AIAA 2009-6257, August 2009.

[24] Koolstra, H. J., Damveld, H. J., and Mulder, J. A., Envelope determination of damaged aircraft, Guidance, Navigation, and Control and Co-located Conferences, No. AIAA 2012-4699, American Institute of Aeronautics and Astronautics, Aug. 2012.

[25] Looye, G. and Bennani, S., Description and Analysis of the Research Civil Aircraft Model (RCAM), Technical report TP-088-27, Group for Aeronautical Research and Technology in Europe GARTEUR, 1997. 